\title{
Accelerating the Knowledge Innovation Process
}

\author{
Guillermo Cortes Robles, Giner Alor Hernández, Alberto Aguilar Lasserre, \\ and Rubén Posada Gómez
}

Instituto Tecnológico de Orizaba, Postgraduate Department,

Av. Oriente 9 No. 852, 94300 Orizaba, Ver. México

\{gcortes, aguilar, galor, rposada\}@itorizaba.edu.mx

\begin{abstract}
The generation of ideas or new concepts is the steppingstone of the innovation process. Nevertheless the transformation of those ideas in new or improved products, services or processes demands the mobilization of a huge diversity of knowledge. In this document is proposed the integration of the Theory of Inventive Problem Solving (TRIZ) and the Case-Based Reasoning (CBR) process in order to conceive a solving process capable to guide creativity while generating innovative solutions and also to store, index and reuse knowledge with the aim to accelerate the innovation process.
\end{abstract}

Keywords: Innovation, TRIZ, CBR, Knowledge, Problem Solving Process.

\section{Introduction}

Innovation has become the main source of value and competitiveness in nowadays market. This topic has been studied in several surveys that have explored the challenges to face when trying to innovate [1], [2]. It is then natural the effort that enterprises have dedicated to manage this complex process in order to improve its performance. This effort involves the development of organizational structures and tools for capturing and transforming ideas, concepts and knowledge in new or improved products, services or processes. It also includes the creation of technical tools to minimize time-to-market, to reduce cost of new products development and to mobilize efficiently the available knowledge within and outside the enterprise frontiers. Therefore, a structure that enables idea generation to solve the problems related to new products development but also capable to capture, store and reuse knowledge as a mean to accelerate the innovation process is highly desirable. In this document is proposed the integration of the Case-Based Reasoning process and the TRIZ theory as a tool for supporting idea generation and knowledge reutilization with the aim to support the innovation process.

Several are the reasons that impel this integration:

(1) Because knowledge must solve problems, not chance. Different from other techniques that assist idea generation, the TRIZ theory is a knowledge-based approach for problem solving that had capitalized knowledge from a vast variety of technical domains. This knowledge has been arranged in such a way that it is available when solving inventive or innovative problems. This condition produces an environment where is possible to transfer strategies, principles and problem solving heuristics that 
have proved its efficacy in other domains in order to increase the efficiency of the problem solving process [3]. (2) TRIZ mobilizes knowledge in a high level of abstraction, nevertheless an approach capable to store, index and reuse specific knowledge it is also need it. The Case-Based Reasoning approach possesses those abilities [4]. (3) TRIZ and CBR are both emulating a central human problem solving process: analogical thinking. This is according numerous authors the intrinsic human process for problem solving [5], [6].

The integration of several TRIZ concepts and the Case-Based Reasoning (CBR) is analyzed in the next three sections: first and second section briefly introduces the TRIZ theory and the Case-Based Reasoning approach, with the aim to show its complementarily. In third section a succinct description of the synergy is presented to finally describe a solved case.

\section{The TRIZ Theory}

The TRIZ theory (Russian acronym for "Theory of Inventive Problem Solving") has been conceived to generate solution avoiding tradeoff. This approach ${ }^{1}$ for problem solving has its origins in the former USSR, where it was founded by G. Altshuller and other scientists [3]. One of the main advantages of TRIZ is that the solution space is not explored randomly. The TRIZ toolbox gives some directions that should be explored in order to derive a solution (the tool that should be applied depends on the nature of the problem). Consequently, TRIZ has the capacity to restrict the research space for innovative solutions and to guide thinking towards solutions or strategies that have demonstrated their efficacy in a past similar situation, besides TRIZ produces an environment where the generation of a potential solution is almost systematic [6]. Of course TRIZ does not give a "ready to use" solution but it proposes some vectors that direct the search for finding innovative solutions, then it leaves place to the designer creativity. Four areas were analyzed to establish the foundations of TRIZ: (1) The global patents database (more than 3 millions of patents have been analyzed). (2) The analysis of scientific literature. (3) The analysis of psychological behavior of inventors and (4) Analysis of existing methods and tools for problem solving.

This analysis revealed the cornerstones of TRIZ that are enclosed in a set of concepts and tools that helps to solve non-routine problems or inventive problems. These concepts and tools give access to the best practices in the whole technical domain thus increasing the creative potential of designer. Among the most important conclusions and TRIZ concepts are next:

1. Problems and solutions were repeated across industries and sciences.

2. Innovations used scientific effects outside the field where they were developed.

3. A set of evolution patterns for technical systems exists. During its life cycle, a system is always evolving and this evolution is governed by objective laws. Thus, knowledge about those patterns is useful to foretell next stages of a product or technology.

4. Patterns of technical evolution were repeated across industries and sciences.

${ }^{1}$ TRIZ has been classified as a theory, methodology, tool, a set of heuristics, etc. 
5. Ideality is a goal in every system. All systems evolve towards the increase of their degree of ideality. One way to measure the ideality is to use the Ideal Final Result (IFR) which is a psychological concept that allows finding the best solution for a complex problem, without taking into account cost, time, space or any problem constraints. This ideal system is often a utopian system but it guides reflection toward rarely explored directions.

6. In TRIZ, problems can be formulated in terms of contradiction. An inventive problem contains at least one contradiction, and an inventive solution overcomes totally or partially this contradiction. Several types of contradictions have been identified, but in this document only physical and technical are defined. Technical contradictions exist when any tentative to improve the performance of a useful function or characteristic in a system, produces an unacceptable deterioration in a second useful function in the system. It represents a conflict between two subsystems or characteristics. A physical contradiction occurs when a component or element in a system demands simultaneously two mutually exclusive states: a surface must be smooth and rough. It represents a conflict in the same subsystem. Contrary to classical methods for creativity stimulation (brainstorming, trial and errors, etc.), TRIZ refuses trade-off and tries to eradicate the contradiction.

Next section introduces the second component in the synergy: the Case-Based Reasoning.

\section{The Case-Based Reasoning (CBR) Process}

Artificial intelligence and more precisely knowledge management approaches try to use past experiences in a domain to solve new problems. The main difficulty is to find a way to store, retrieve and reuse knowledge inside an enterprise but also, to define the mechanisms to filter the knowledge in the surrounding enterprise environment. Coming from AI, CBR is a very useful approach to manage knowledge. The main idea in CBR is that similar problems have similar solutions. Basically in the CBR process users try to solve a new problem by establishing similar patterns between the initial problem and some previous experiences (solved problems). Then the CBR process uses and adapts earlier successful (or failed) solutions in order to solve the new problem. This process is at the core of everyday human problem solving.

The CBR method is a cyclic process involving at least five stages: represent, retrieve, reuse, revise and retain. The first stage or representation consists only in obtaining the relevant features that characterized a problem (i.e. components, apparatus, flow rates, pressure, temperature, etc.). With this information is possible to start the retrieving stage in which the problem to solve is compared with the cases stored in the memory with the aim to identify the most similar. Then if one or various stored cases match with the target problem, the most similar case is selected to reuse its solution. Subsequently, the derived solution must be revised, tested and repaired if necessary to increase the possibilities to obtain a satisfactory result or to avoid failure. Finally the new experiences which comprise failure or success, but also the strategies to repair and implement the final solutions (among other particular features), are retained for further utilization and the previous case memory is updated [7].

The inherent process of the CBR reduces time when solving problems because it gives an initial model for deriving solutions. Usually, it is more efficient to solve a 
problem from an existing starting point than to develop the whole solution from nothing. For a good performance of a CBR system, the case base must cover the whole or an important part of the problem space (all the problems that may appear in the specific domain of application). Consequently, the efficacy of the systems relies on the structure, quantity and quality of the stored cases.

Both approaches are useful to solve problems, nevertheless their objectives are different. Among the main differences between TRIZ and CBR are:

Table 1. Differences between TRIZ and CBR

\begin{tabular}{|l|l|}
\hline \multicolumn{1}{|c|}{ CBR } & \multicolumn{1}{c|}{ TRIZ } \\
\hline $\begin{array}{l}\text { Limited to a specific domain (specificity } \\
\text { could be a barrier to creativity) }\end{array}$ & $\begin{array}{l}\text { Transversal application (all technical fields), } \\
\text { an environment to stimulate creativity }\end{array}$ \\
\hline Routine design & Inventive design \\
\hline $\begin{array}{l}\text { No solution if the initial problem can not find } \\
\text { a similar case }\end{array}$ & Gives a way of solution for each problem \\
\hline Produces a solution from an initial model & Produces a solution starting from "nothing" \\
\hline $\begin{array}{l}\text { Posses a memory: solutions are produced } \\
\text { rapidly. System become more efficient by } \\
\text { learning }\end{array}$ & $\begin{array}{l}\text { No memory; resolution process redeployed } \\
\text { each time }\end{array}$ \\
\hline $\begin{array}{l}\text { Easy for use, thanks to its affinity with human } \\
\text { resolution process }\end{array}$ & $\begin{array}{l}\text { Difficult to use because of its particular way } \\
\text { to tackle problems and its variety of tools }\end{array}$ \\
\hline
\end{tabular}

Once described the differences and complementarily between both approaches, it is possible to describe the integration of TRIZ and CBR.

\section{The TRIZ-CBR Integration}

Altshuller discovered that very different technical systems and processes share similarities in their evolutions. For example the same generic problem had been pointed out and solved with the same generic principle of resolution but in different technical domains and sometimes the solutions were separated by many years. Consequently, Altshuller thought that if inventors or engineers can benefit from successful solutions found in others disciplines, the innovation process will be more efficient [8]. A tool called contradiction matrix crystallize this point of view. During the patents analysis, Altshuller notices that technical contradictions can be expressed in terms of conflict between two parameters (with a limited number of parameters): one improved and the other one damaged. Only 39 parameters were extracted to describe all the contradictions encountered in patents. Representing technical contradiction as a combination of two parameters requires a broad interpretation of them, so they are generic for many engineering fields. Finally, a $39 \times 39$ matrix was built. On the line, is located the improved parameter, on the column the damaged one or the parameter that prohibit an improvement. For one contradiction, the cell at the intersection of the line and the column indicates the principle(s) to explore in order to solve it. This matrix was updated in 2003 and the number of parameters was increased to 48.

Through the contradiction matrix, TRIZ opens up the world patents bases for identifying principles that may offer possible solutions. Based on the advantages previously mentioned, the contradiction matrix was transformed in the case memory for the model schematized in next figure: 


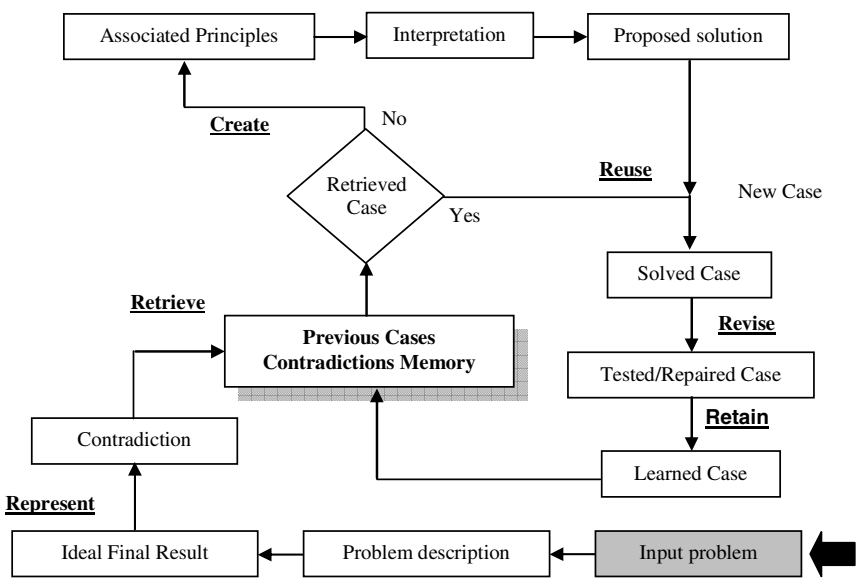

Fig. 1. The TRIZ-CBR solving problem process

The solving process starts whit the formulation and representation of the problem to solve. The process demands at least four initial features concerning the problem description: (1) the system where the problem is located; (2) the type of problem or objective: reduction/elimination of harmful function, improvement of a characteristic or new functionality; (3) the goal to reach; (4) the resources identified in the system. After this step, the ideal solution is also stated in order to offer a guide for the search direction of the future solution. Then the problem is stated as a contradiction to obtain a more robust problem description. With those data (contradiction and the other features) is possible to explore the memory and search for a similar problem. At this point of the synergy process, two different sub processes can take place:

1) The retrieval offers a similar problem or set of problems. The most similar is selected to adapt its solution (to be used as initial solution). Here the similarity between two problems is calculated with a similarity global function:

$$
S I M=\frac{\sum_{i=1}^{n} w_{i} * \operatorname{sim}\left(f_{i}^{l}, f_{i}^{R}\right)}{\sum_{i=1}^{n} w_{i}}
$$

Where $f_{i}^{I}, f_{i}^{R}$ represent respectively the features $i$ for the initial problem (I) and the retrieved cases (R), sim the local similarity function for this feature $i$ and $w_{i}$ the weight of the feature $i$. [9] discuss the different manners to measure local similarity, it depends of the type of feature value: semantic, symbolic, numeric.... SIM represents the global similarity. If various similar cases are found, the global similarity function ranks them. Moreover, the global similarity function can be customised thanks to the weight, in order to give more importance to one feature to others, which is the case of the tool presented in next section. When calculating similarity, technical contradiction is the most important factor, then the available resources in the system followed by the type of problem and the goal to reach. 
2) The memory does not have any similar solved case or sufficiently similar case (the similarity global function has a too small value). Under this condition, the system offers inventive principles associated to the contradiction, by which a satisfactory solution could be derived. The matrix finds its initial use [10].

The process exposed in figure 2 is the basis for a tool that helps to solve problems stated as a technical contradiction (this model was tested with more than 100 patents). This tool has two different processes: first one is useful to load problems in the case memory. All the cases indexed and stored in the memory are evaluated by an expert(s) to decide about its pertinence. Second process facilitates the search in the case memory. In next example is shown a case that does not have a similar case in the memory.

\section{A Solved Case: Proposing a New Product}

A local SME offers us the opportunity to develop a new product. The enterprise was searching ideas for developing a totally new "outside living set" line. To deal with this problem a five stages process was deployed. Stage (1) was the recognition of costumer's needs. This information was gathered -locally- from clients and other similar products. International information was also collected. Stage (2) concerns problem definition. Available information about costumer's needs was the basis for defining "the design problem" which encompasses materials, production means, ergonomic specifications and marketing among other design stakeholders. Stage (3) affects concepts development that ideally should satisfy all product dimensions. Stage (4) involves concept validation. An expert panel was responsible to evaluate prototypes and to select the most promising product. Stage (5) transforms the selected concept in product specifications and production requirements.

In this document are described only stages 2 and 3 in order to show how the problem was undertaken.

- System description: an outside living set include at minimum one table and four chairs. The main useful function is to offer a surface for resting and supporting objects. The product is available in a high diversity of materials and shapes. Next figures show typical products:
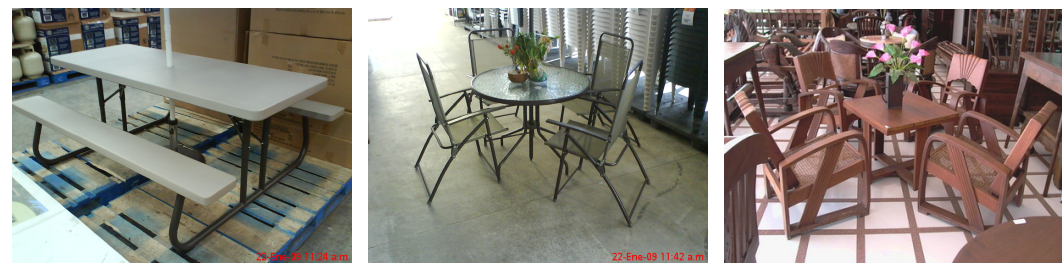

Fig. 2. Typical products

- Problem statement: Costumer's information reveals that the living set should be adaptable, lightweight -easy to move-, resistant, easy to clean, aesthetically attractive, etc. But according the enterprise perspective, the most important feature is that should be different from similar products available in the market. Then it is necessary to propose a concept enclosing all those characteristics. 
- The solution must satisfy next restrictions:

(1) To be lightweight, (2) Maximal mechanical resistance (no more than $110 \mathrm{~kg}$ ),

(3) Cost should not exceed the typical price of available products, (4) Easy to move, clean and remove when is not in use, (5) The selected prototype should be produced with the available means.

- IFR (Ideal Final Result or more desirable result): one of the costumers offer us next requirement "the living set should be there only when I need it and disappear the rest of the time". Reusing and complementing this description: the living set satisfies all costumer requirements and is available only when he/she needs it.

- Resources in the system:

\begin{tabular}{|l|l|}
\hline Substance proprieties & $\begin{array}{l}\text { Metal: conductivity, rigidity, mechanical resistance, } \\
\text { thermal expansion, among others. }\end{array}$ \\
\hline & $\begin{array}{l}\text { Plastic: flexibility, lightweight, weather resistance, non- } \\
\text { expensive, among others. }\end{array}$ \\
\hline & Wood: rigidity, density, humidity, among others. \\
\hline Shape & Space, void, among others. \\
\hline Available fields: thermal, gravitational.
\end{tabular}

- Contradictions: numerous contradictions were identified and arranged hierarchically. The first contradiction to solve was stability versus shape (this decision was taken by the team using the analytical hierarchy process). The associated principles to this contradiction are: 1 (segmentation), 4 (Symmetry change), 35 (Parameter changes), 17 (Dimensionality change), 7 (Nested doll) and 3 (Local quality).

- Deriving solutions from inventive principles. Principle 1 suggests "to divide an object or system into independent parts". The set is already fractionated. Principle 4 proposes "Change the shape of an object or system from symmetrical to asymmetrical". Principle 35 recommends "Change an object's physical state. Change the concentration or consistency. Change the degree of flexibility" any potential solution was obtained from those principles.

- Principle 17 suggests "Move an object or system in two- or three-dimensional space. Use a multistory arrangement of objects instead of a single-story arrangement. Tilt or reorient the object, lay it on its side, use its other side" and principle 7 proposes "Place one object inside another; place each object, in turn, inside the other. Make one part pass through a cavity in the other". Principle 17 and 7 guide the search for a conceptual solution. The resource utilized to materialize this concept was existing space.

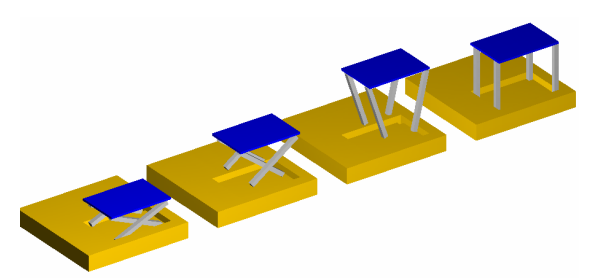

Fig. 3. Proposed solution 
This concept was the basis for several prototypes. The idea is to make the living set easy to assemble/disassemble in such a way that costumers will have the living set only when it is necessary.
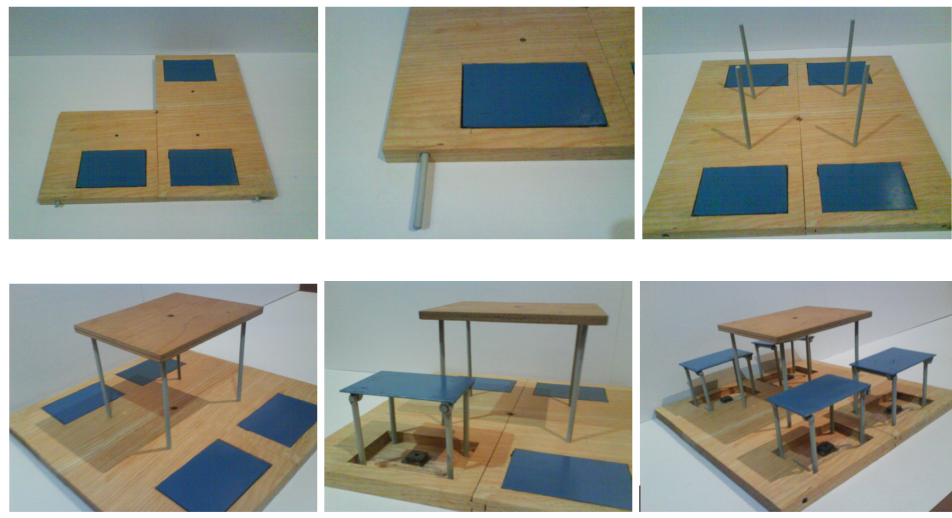

Fig. 4. Outside living set

This was the first prototype. This concept was transformed by using the evolution patterns proposing a new variety of products. The enterprise conserves those ideas.

\section{Conclusions}

The presented model offers a vector to transfer the solution from an identified analogous problem into a new target problem, reducing effort and time when solving inventive problems. The model also has a tool to guide creativity when there is not a case stored in the memory and the user needs to generate a completely new solution. The model combines the TRIZ ability to apply general knowledge in a very creative way and a framework that closely relates knowledge and action. But maybe the most important benefit of this model is that the model assists the learning process. The case memory transforms in a reusable way the experiences obtained while solving problems. This condition produces an environment that impulse knowledge sharing. The model exposed conserves the capacities and advantages of both components and minimize the identified disadvantages.

The main drawback of the model it is the identification of the right contradiction. The formulation of contradictions is not an exact process, is a subjective activity strongly influenced by previous experiences and knowledge. Thus, in the model it's necessary to assist users to accomplish this stage.

\section{Acknowledgement}

This work is supported by the General Council of Superior Technological Education of Mexico (DGEST). Additionally, this work is sponsored by the National Council of 
Science and Technology (CONACYT) and the Public Education Secretary (SEP) through PROMEP.

\section{References}

1. Rowell A.: The Innovator's Toolbox. Aberdeen Group, (2009), http: / / www . aberdeen. com/summary/report/benchmark/ 5381-RA-empowering-difference-makers.asp

2. Andrew J., Haanaes K., Michael D., Sirkin H., Taylor A.: Measuring Innovation. The Boston Consulting Group (2008), http://www.bcg.com/impact_expertise/publications/files/ Measuring_Innovation_Aug_2008.pdf

3. Altshuller, G.: Creativity as an exact science: The theory of the solution of inventive problems. Gordon and Breach Publishers (fourth printing) (1998)

4. Watson, I.: Applying Case-Based Reasoning: Techniques for enterprise systems. Morgan Kaufmann, San Francisco (1997)

5. Nonaka, I., Takeuchi, H.: La connaissance créatrice. La dynamique de l'entreprise apprenante. De Boeck Université (1997)

6. Terninko, J., Zusman, A., Zotlin, B.: Systematic Innovation: An Introduction to TRIZ. St. Lucie Press (1998)

7. Pal, S., Shiu, S.: Foundations of soft Case-Based Reasoning. John Wiley \& Sons Publication, Chichester (2004)

8. Altshuller, G.: The Innovation Algorithm. Technical Innovation Center (1999)

9. Avramenko, Y., Kraslawski, A.: Similarity concept for case-based design in process engineering. Computers \& Chemical Engineering 30, 548-557 (2006)

10. Cortes Robles, G., Negny, S., LeLann, J.M.: Case-based reasoning and TRIZ: A coupling for innovative conception in Chemical Engineering. In: Chemical Engineering and Processing: Process Intensification. Elsevier, Amsterdam (2008) 